DAMTP-R/94/16

\title{
CONFIGURATIONS OF HANDLES AND THE CLASSIFICATION OF DIVERGENCES IN THE STRING PARTITION FUNCTION
}

\author{
Simon Davis \\ Research Foundation of Southern California \\ 5580 La Jolla Boulevard, La Jolla, CA 92037 \\ and \\ Department of Applied Mathematics and Theoretical Physics \\ University of Cambridge \\ Silver Street, Cambridge CB3 9EW*
}

\begin{abstract}
The divergences that arise in the regularized partition function for closed bosonic string theory in flat space lead to three types of perturbation series expansions, distinguished by their genus dependence. This classification of infinities can be traced to geometrical characteristics of the string worldsheet. Some categories of divergences may be eliminated in string theories formulated on compact curved manifolds.
\end{abstract}

* Present address 


\section{Introduction}

The resolution of fundamental problems in string theory such as the determination of the stability of phenomenologically realistic vacua and the existence of black hole solutions, might require a complete formulation of the theory including non-perturbative effects as well as S-matrix calculations [1][2]. A study of the perturbation series, however, may be sufficient to establish stability of the vacuum. For closed bosonic strings moving in 26-dimensional flat space-time, the infrared divergences in the partition function can be eliminated through integration over a subset of moduli space that does not include the degeneration locus. Although an estimate has already been made for the genus-dependent growth of integrals with this cut-off over Teichmuller space, it will be altered by the restriction from Teichmuller space to moduli space [3]. A precise definition of the integration region is necessary to establish rigorously that the flat background is not stable against perturbations.

Through an analysis of the conditions defining the fundamental region of the modular and mapping class groups, a lower bound for the regularized partition function has been found [3][5]. While the action of the symplectic modular group $\operatorname{Sp}(2 \mathrm{~g} ; \mathbb{Z})$ is defined initially on a $\frac{1}{2} \mathrm{~g}(\mathrm{~g}+1)$-dimensional space of positive-definite symmetric matrices, the fundamental domain can be projected onto a (3g-3)-dimensional subspace of period matrices $\tau$ for Riemann surfaces of genus g satisfying the inequalities

$$
\begin{aligned}
& \text { (i) } \quad-\frac{1}{2} \leq \operatorname{Re} \tau_{m n} \leq \frac{1}{2} \\
& \text { (ii) }|\operatorname{det}(C \tau+D)| \geq 1 \quad\left(\begin{array}{ll}
A & B \\
C & D
\end{array}\right) \in \operatorname{Sp}(2 g ; \mathbb{Z}) \\
& \text { (iii) } \operatorname{Im} \tau\left[g_{r}\right]=g_{r}^{T}(\operatorname{Im} \tau) g_{r} \geq \operatorname{Im} \tau\left[e_{r}\right] \text { where } e_{r}=(0, \ldots, 0,1,0, \ldots, 0) \\
& \quad \text { and } g_{r}=\left(g_{r 1}, \ldots, g_{r r}, \ldots, g_{r g}\right) \text { where } g_{r r}, \ldots, g_{r g} \text { are relatively } \\
& \quad \text { prime; }(\operatorname{Im} \tau)_{1 r} \geq 0
\end{aligned}
$$

The bound for the partition function has been obtained, using another set of variables, the multipliers $\left\{K_{n}\right\}$ and the fixed points $\left\{\xi_{1 n}, \xi_{2 n}\right\}$, characterizing the generators $\left\{T_{n}, n=\right.$ $1, \ldots g\}$ of the Schottky group uniformizing the Riemann surface. In particular, limits have 
been placed on the multipliers and fixed points using the relation between the elements of the period matrix and these variables [4],

$$
\tau_{m n}=\frac{1}{2 \pi i}\left[\ln K_{m} \delta_{m n}+\sum_{\alpha}{ }^{(m, n)} \ln \frac{\xi_{1 m}-V_{\alpha} \xi_{1 n}}{\xi_{1 m}-V_{\alpha} \xi_{2 n}} \frac{\xi_{2 m}-V_{\alpha} \xi_{2 n}}{\xi_{2 m}-V_{\alpha} \xi_{1 n}}\right]
$$

with $V_{\alpha}$ being a product of $T_{n_{i}}$ and $\sum_{\alpha}{ }^{(m, n)}$ excluding all $V_{\alpha}$ containing $T_{m}^{ \pm 1}$ at the left and $T_{n}^{ \pm 1}$ at the right.

The vacuum amplitudes at finite order may be factored out of the higher-point correlation functions as the closed Riemann surfaces are equivalent to the bubble diagrams of field theory. Because of the factorization of the closed string vacuum amplitude, the physical significance of the cut-off integral over moduli space is revealed in the effect of the summability of the series on the stability of the classical background. Moreover, it allows one to study the limit as the number of handles tends to infinity, where the surfaces, depending on the classification type, may represent the creation of a point-particle or string state from the vacuum. The four-point function on an infinite-genus surface of type $O_{G}$ has been evaluated in previous work [6].

Surfaces containing an infinite number of accumulating handles affect the Borel summability of the perturbation series. It will be demonstrated that there are three categories of surfaces associated with distinct arrangements of the isometric circles $\left\{I_{T_{n}}\right\}$ of the generators in the complex plane. The growth of both the lower and upper bounds is related to the geometrical characteristics of the configuration of isometric circles or equivalently the handles on the surfaces.

For strings propagating in flat space-time, the growth of the bound for the partition function is obtained after an analysis of each of the configurations of isometric circles which lie in the fundamental region of the symplectic modular group [7]. The three categories considered are determined by the the dependence of the multipliers and fixed-point distances on the genus, which are related because $\frac{\left|K_{n}\right|}{\left|1-K_{n}\right|^{2}}=\frac{\left|\gamma_{n}\right|^{-2}}{\left|\xi_{1 n}-\xi_{2 n}\right|^{2}}$ and $\min _{n}\left|\gamma_{n}\right|^{-2} \sim \frac{1}{g}$ as a consequence of requiring genus-independence of the cut-off [9]. If $\left|\xi_{1 n}-\xi_{2 n}\right| \sim \frac{1}{g^{q}}$, the values $\mathrm{q}=0, q=\frac{1}{2}$, and $0<q<\frac{1}{2}$ define the three types of configurations. It is demonstrated 
in this paper that the contributions of the correponding surfaces to the string partition function will increase as $k^{g} \frac{g^{g}}{(\ln g)^{13 g}}, k^{\prime g}\left(\frac{g}{J}\right)$ ! and $k^{\prime \prime g}\left(\frac{g^{g}}{(\ln g)^{13 g}}\right)$ respectively. These estimates follow from an evaluation of upper and lower bounds for the integrals with limits for the Schottky group parameters corresponding to the different values of q. This establishes rigorously that an approximately factorial growth will occur in the regularized string partition function, a property found in previous work [2][3] for the string integral over subset of Teichmuller space without restriction to the fundamental domain of the mapping class group.

Since each of the terms in the perturbation series is positive, being the volume of a region in a (3g-3)-dimensional parameter space, an exactly factorial increase, $k^{g} g$ ! would indicate that the series is not Borel summable. The growth obtained here for specific values of $\mathrm{q}$ is not exactly factorial, so that its effect on the Borel summability of the series would require further analysis. For the three categories of isometric circles studied here, the modified series, defined by dividing the gth term by g!, would be convergent on the entire positive real axis in the coupling constant plane, allowing for the use of the Borel integral transform. However, summation of the contributions of the three kinds of configurations to the partition function only comprises part of the regularized moduli space integral. Specifically, the third category can be subdivided into a further $O(\ln g)$ sub-categories, and by considering configurations of isometric circles with multipliers $\left\{K_{n}\right\}$ having different genus dependence for different $n$, an additional combinatorial factor of the form $\frac{(g+k \ln g-1) !}{g !(k \ln g-1) !}$ is introduced. While this improves the rapidly increasing growth of the bounds for the regularized integral, it is still not large enough to alter the Borel summability properties of the series. Additional contributions to the string partition function arise from special configurations within the three categories that lead to a change in the genusdependence of certain factors in the moduli space integral. By applying the techniques used here to these configurations, the magnitude of the contributions may be estimated, and it appears that they will not significantly change the bounds. Finally, one might investigate other categories of isometric circles consistent with a genus-independent cut-off on the length of closed geodesics on the surface. A discussion of a separate category of 
isometric circles is given in Section 3, and a complete study of the other configurations may be required to find a precise estimate of the regularized string integral in the Schottky group parametrization.

The results obtained in this paper indicate that it may be useful to re-examine the application of graph theory [3], used to obtain a factorial growth with respect to the genus, in the large-order limit. In particular, it is suggested that a there is a potential difference between the counting of diagrams corresponding to string and point-particle trajectories and this would be determined by the physical intepretation of string theory in the large-genus limit.

The change of metric in a curved manifold leads to a different bosonic string path integral. The divergences in the partition function for strings propagating in a curved background are considered, and an outline of the analysis of the allowed configurations of isometric circles in a compact target space and their effect on Borel summability is presented.

\section{Divergences in Flat Space Closed Bosonic String Perturbation Theory}

By integrating over metrics on the string worldsheet and target space coordinates in $\mathbb{R}^{26}$, the partition function for the closed string theory, obtained by summing over the genus,

$$
Z=\sum_{g=0}^{\infty} \kappa^{g} \int \frac{D\left[h_{\alpha \beta}\right] D\left[X_{\mu}\right]}{\operatorname{Vol}\left(\operatorname{Diff} \Sigma_{g}\right) \operatorname{Vol}\left(\operatorname{Conf} \Sigma_{g}\right)} e^{-\frac{1}{2} \int d^{2} \xi \sqrt{h} h^{\alpha \beta} \partial_{\alpha} X^{\mu} \partial_{\beta} X_{\mu}}
$$

can be re-expressed in terms of a measure involving Schottky group variables $K_{n}, \xi_{1 n}$ and $\xi_{2 n}$, which are the multipliers and fixed points defining the generating transformations of the uniformizing group through $\frac{T_{n} z-\xi_{1 n}}{T_{n} z-\xi_{2 n}}=K_{n} \frac{z-\xi_{1 n}}{z-\xi_{2 n}}$. The partition function [8] is given 
by

$$
\begin{array}{r}
Z=\sum_{g=0}^{\infty} c_{g} \kappa^{g} \int_{F_{g}} \prod_{m=1}^{g} \frac{d^{2} K_{m}}{\left|K_{m}\right|^{4}}\left|1-K_{m}\right|^{4} \frac{1}{V o l(S L(2, \mathbb{C}))} \prod_{n=1}^{g} \frac{d^{2} \xi_{1 n} d^{2} \xi_{2 n}}{\left|\xi_{1 n}-\xi_{2 n}\right|^{4}} \\
\cdot[\operatorname{det}(\operatorname{Im} \tau)]^{-13} \prod_{\alpha}^{\prime} \prod_{p=1}^{\infty}\left|1-K_{\alpha}^{p}\right|^{-48} \prod_{\alpha}^{\prime}\left|1-K_{\alpha}\right|^{-4}
\end{array}
$$

where $\tau$ is the period matrix, $K_{\alpha}$ is the multiplier of the element $V_{\alpha}, \prod_{\alpha}^{\prime}$ represents the product over conjugacy classes of primitive elements, and $F_{g}$ is the fundamental region of the modular group in the parameter space. In earlier work [5], a lower bound was set for each of the terms in the integrand in equation (2), when the Riemann surface $\Sigma_{g}$ is constrained by the requirement that the minimal length of closed geodesics on the manifold be bounded below.

The action of the generator $T_{n}, T_{n} z=\frac{\alpha_{n} z+\beta_{n}}{\gamma_{n} z+\delta_{n}}$, on the complex plane involves the mapping of the exterior of $I_{T_{n}}=\left\{z \in \mathbb{C}|| \gamma_{n} z+\delta_{n} \mid=1\right\}$ to the the interior of $I_{T_{n}^{-1}}=$ $\left\{z \in \mathbb{C}|| \gamma_{n} z-\alpha_{n} \mid=1\right\}$ and the interior of $I_{T_{n}}$ to the exterior of $I_{T_{n}^{-1}}$. There are three types of configurations of isometric circles determined by the behaviour of the multipliers and fixed points consistent with the cut-off $\left|\gamma_{n}\right|^{-2} \sim \frac{1}{g}$. Other configurations of isometric circles consistent with this decrease in the radii of circles on a sphere of projection are considered in the following section. The three categories of isometric circles studied in this section are characterized by the dependence of the limits on the genus.

$$
\begin{array}{ll}
\text { (i) } \frac{c_{1}}{g} \leq\left|K_{n}\right| \leq \frac{c_{2}}{g} & \delta_{0} \leq\left|\xi_{1 n}-\xi_{2 n}\right| \leq \delta_{0}^{\prime} \\
\text { (ii) } \quad \epsilon_{0} \leq\left|K_{n}\right| \leq \epsilon_{0}^{\prime} & \frac{\delta_{0}}{\sqrt{g}}+d\left(\xi_{1 n}, I_{T_{n}^{-1}}\right) \leq\left|\xi_{1 n}-\xi_{2 n}\right| \leq \frac{\delta_{0}^{\prime}}{\sqrt{g}} \\
\text { (iii) } \frac{\epsilon_{0}}{g^{1-2 q}} \leq\left|K_{n}\right| \leq \frac{\epsilon_{0}^{\prime}}{g^{1-2 q}} & \frac{\delta_{0}}{g^{q}}+d\left(\xi_{1 n}, I_{T_{n}^{-1}}\right) \leq\left|\xi_{1 n}-\xi_{2 n}\right| \leq \frac{\delta_{0}^{\prime}}{g^{q}} \quad 0<q<\frac{1}{2}
\end{array}
$$

Bounds for the integrals over subsets of moduli space corresponding to the first two types of configurations have been found [5]. In the following analysis, these bounds are refined and an estimate of the contribution of the third type of configuration is given.

By setting an upper bound on $\sum_{\alpha}{ }^{\prime}\left|K_{\alpha}\right|=\sum_{\alpha}{ }^{\prime} \frac{\left|\gamma_{\alpha}\right|^{-2}}{\left|\xi_{1 \alpha}+\frac{\delta_{\alpha}}{\gamma_{\alpha}}\right|^{2}}$, the next two inequalities have been obtained for isometric circles defined by the limits in category (i). 


$$
\begin{aligned}
\prod_{\alpha}{ }^{\prime} \prod_{p=1}^{\infty}\left|1-K_{\alpha}^{p}\right|^{-48} \prod_{\alpha}{ }^{\prime}\left|1-K_{\alpha}\right|^{-4}> & \exp \left(\frac{-192 c^{4}}{c^{2}-2 c_{2}} \frac{d_{\max }^{2}\left(\left\{I_{T_{n}}, I_{T_{n}^{-1}}\right\}\right)}{d_{\min }^{2}\left(\left\{I_{T_{m}}\right\}\right)}\right) \\
& \left.\left(\frac{1}{2}\right)^{\left(1+\frac{192}{c_{2}} \frac{d_{\max }^{2}\left(\left\{I_{T_{n}}, I_{T_{n}}^{-1}\right\}\right)}{d_{\min }^{2}\left(\left\{I_{T_{m}}\right\}\right)}\left(\left(c_{2}-c^{2}-c+\frac{1}{2}\right) C_{0}-C_{1}\right)\right.}\right)
\end{aligned}
$$

$$
\begin{aligned}
& C_{0}=\frac{c^{4}}{c^{2}-2 c_{2}} \\
& C_{1}=-c_{2} c^{3} \frac{\left(c-4 c_{2}-4 c_{2} c\right)}{\left(c^{2}-2 c_{2}\right)^{2}} \\
& C_{2}=\frac{c^{2}(1+c) c_{2}^{2}}{c^{2}-2 c_{2}}+\frac{2 c^{3}(1+c) c_{2}^{2}-5 c^{4}(1+c)^{2} c_{2}^{2}}{\left(c^{2}-2 c_{2}\right)^{2}}+\frac{c^{4} c_{2}^{2}(1-2 c(1+c))^{2}}{\left(c^{2}-2 c_{2}\right)^{3}}
\end{aligned}
$$

with c being a lower bound for $\left|\frac{\xi_{2 n}-\frac{\alpha_{\beta}}{\gamma_{\beta}}}{\xi_{2 n}-\xi_{1 n}}\right|$ for bounded g, when the distance between the isometric circles is $\mathrm{O}(1)$, and a genus-independent constant for large g, $I_{V_{\beta}^{-1}} \not \subset D_{T_{n}}=$ $\left\{z|| \gamma_{n} z+\delta_{n} \mid \leq 1\right\}, d_{\min }\left(\left\{I_{T_{m}}\right\}\right)$ equal to the minimum distance between the isometric circles and $d_{\max }\left(\left\{I_{T_{n}}, I_{T_{n}^{-1}}\right\}\right)$ equal to the maximum distance between $I_{T_{n}}$ and $I_{T_{n}^{-1}}$, for all n;

$$
[\operatorname{det}(\operatorname{Im} \tau)]^{-13}>(2 \pi)^{13 g}\left[\ln g+\frac{8 c}{c^{2}-2 c_{2}}-\ln c_{1}\right]^{-13 g}
$$

Configurations of isometric circles in each of the three categories satisfy a set of conditions defining a fundamental region of the symplectic modular group acting on a (3g-3)dimensional subset of the Siegel upper-half space if appropriate restrictions are placed on the multipliers $K_{n}$ and fixed points $\xi_{1 n}$ and $\xi_{2 n}$. The fundamental set of inequalities result from $\operatorname{Im} \tau\left[g_{r}\right] \geq \operatorname{Im} \tau\left[e_{r}\right]$ when $g_{r}=e_{s}, s \geq r$. It has been shown that these inequalities imply the conditions for a general column vector $g_{r}$ at sufficiently large genus [5]. For isometric circles of the first type, the inequalities $(\operatorname{Im} \tau)_{s s} \geq(\operatorname{Im} \tau)_{r r}, s \geq r$, lead to a 
reduction of the integration range of the multipliers. Denoting

$$
\begin{array}{r}
\rho^{-1}=\max _{n} \prod_{\alpha}(n, n)\left|1-\frac{\gamma_{\alpha}^{-2}\left(\xi_{1 n}-\xi_{2 n}\right)^{2}}{\left(\xi_{1 n}+\frac{\delta_{\alpha}}{\gamma_{\alpha}}\right)\left(\xi_{2 n}+\frac{\delta_{\alpha}}{\gamma_{\alpha}}\right)\left(\xi_{1 n}-V_{\alpha} \xi_{1 n}\right)\left(\xi_{2 n}-V_{\alpha} \xi_{2 n}\right)}\right| \\
\sigma=\min _{n} \prod_{\alpha}(n, n)\left|1+\frac{\gamma_{\alpha}^{-2}\left(\xi_{1 n}-\xi_{2 n}\right)^{2}}{\left(\xi_{1 n}+\frac{\delta_{\alpha}}{\gamma_{\alpha}}\right)\left(\xi_{2 n}+\frac{\delta_{\alpha}}{\gamma_{\alpha}}\right)\left(\xi_{1 n}-V_{\alpha} \xi_{2 n}\right)\left(\xi_{2 n}-V_{\alpha} \xi_{1 n}\right)}\right|
\end{array}
$$

the range of multipliers $K_{1}, \ldots, K_{g}$ can be selected to be $\left[\rho^{-2} \frac{c_{1}}{g}, \sigma^{2} \frac{c_{2}}{g}\right],\left[\rho^{-2} \frac{c_{1}}{g}, \sigma^{2}\left|K_{1}\right|\right]$ $, \ldots,\left[\rho^{-2} \frac{c_{1}}{g}, \sigma^{2}\left|K_{g-2}\right|\right],\left[\frac{c_{1}}{g}, \sigma^{2}\left|K_{g-1}\right|\right]$, so that the integral over the multipliers will be reduced to

$$
\begin{aligned}
&(2 \pi)^{g} \int_{\rho^{-2} \frac{c_{1}}{g}}^{\frac{c_{2}}{g}} \int_{\rho^{-2} \frac{c_{1}}{g}}^{\sigma^{2}\left|K_{1}\right|} \ldots \int_{\rho^{-2} \frac{c_{1}}{g}}^{\sigma^{2}\left|K_{g-2}\right|} \int_{\frac{c_{1}}{g}}^{\sigma^{2}\left|K_{g-1}\right|} \frac{d\left|K_{g}\right|}{\left|K_{g}\right|^{3}} \frac{d\left|K_{g-1}\right|}{\left|K_{g-1}\right|^{3}} \ldots \frac{d\left|K_{2}\right|}{\left|K_{2}\right|^{3}} \frac{d\left|K_{1}\right|}{\left|K_{1}\right|^{3}} \\
&=(2 \pi)^{g} \sum_{j=0}^{g} \kappa_{g-j}(\sigma, \rho)\left[1-\frac{c_{1}^{2}}{\rho^{4} \sigma^{4(g-j)} c_{2}^{2}}\right]^{g-j} \frac{\rho^{4(g-j)} g^{2(g-j)}}{2^{g-j} c_{1}^{2(g-j)}} \\
& \kappa_{g}(\sigma, \rho)=\frac{\sigma^{2 g(2 g-1)}}{g !} \\
& \kappa_{g-1}=0 \\
& \kappa_{g-2}(\sigma, \rho)=-\frac{\sigma^{4} \rho^{8} g^{4}}{c_{1}^{4}}\left[1-\frac{1}{\sigma^{4}}\right]^{2} \frac{\sigma^{2(g-2)(g-3)}}{(g-2) !} \\
& \kappa_{g-3}(\sigma, \rho)=-\frac{\sigma^{12} \rho^{12} g^{6}}{3 ! 8 c_{1}^{6}}\left[1-\frac{1}{\sigma^{8}}\right]^{3} \frac{\sigma^{2(g-3)(g-4)}}{(g-3) !}
\end{aligned}
$$

For a configuration of $2 \mathrm{~g}$ isometric circles involving a minimal spacing $d_{\min }\left(\left\{I_{T_{m}}\right\}\right)=$ $O\left(\frac{1}{\sqrt{g}}\right)$ and contained in a circular region of radius $O(1)$, the ratio $\left|\frac{\xi_{1 n}-\xi_{2 n}}{\xi_{1 n}+\frac{\delta_{T_{l}}}{\gamma_{T_{n}}}}\right|=O(\sqrt{g})$ for isometric circles $I_{T_{n_{l}}}$ near $\xi_{1 n}$, and $\left|\frac{\xi_{1 n}-\xi_{2 n}}{\xi_{1 n}+\frac{\delta_{\alpha}}{\gamma_{\alpha}}}\right|=O(1)$ for circles $I_{V_{\alpha}}$ near the boundary of the region. It follows that the largest remainder terms in $\rho^{-1}$ and $\sigma$ will be of order $O\left(\frac{1}{\sqrt{g}}\right)$, since $\left|\gamma_{n_{l}}\right|^{-2}=O\left(\frac{1}{g}\right)$. However, in a symmetrical configuration of isometric circles about $\xi_{1 n}$, for every circle $I_{T_{n_{l_{1}}}}$ near $\xi_{1 n}$, there may be another circle $I_{T_{n_{l_{2}}}}$ in the same 
neighbourhood lying on the opposite side of $\xi_{1 n}$. If the arguments of $\gamma_{n_{l_{1}}}$ and $\gamma_{n_{l_{2}}}$ are equal, then the product of the two corresponding terms in equation (6) would have the form $1-O\left(\frac{1}{g}\right)$. Since the products in (6) are defined over an infinite number of isometric circles, the phases of $\gamma_{\alpha}^{-2}$ will be randomly distributed in the interval $[-\pi, \pi]$ and many of the terms of order $O\left(\frac{1}{\sqrt{g}}\right)$ may cancel, leaving a remainder term of order $O\left(\frac{1}{g}\right)$. When $\sigma=1-\frac{\sigma_{1}}{g}+\frac{\sigma_{2}}{g^{2}}+\ldots$, it can be demonstrated, that in the limit as $g \rightarrow \infty$, the sum in equation (7) is

$$
\begin{gathered}
e^{-2 \sigma_{1} g} \frac{\rho^{4 g} g^{2 g}}{2^{g} c_{1}^{2 g} g !}\left[e^{2 \sigma_{1}}\left[1-\frac{c_{1}^{2} \sigma^{4} e^{4 \sigma_{1}}}{\rho^{4} c_{2}^{2}}\right]^{g}-8 e^{10 \sigma_{1}} \sigma^{16} \sigma_{1}^{2}\left[1-\frac{c_{1}^{2} \sigma^{12} e^{4 \sigma_{1}}}{\rho^{4} c_{2}^{2}}\right]^{g-2}\right. \\
\left.+\frac{256}{3} e^{14 \sigma_{1}} \sigma^{36} \sigma_{1}^{3}\left[1-\frac{c_{1} \sigma^{16} e^{4 \sigma_{1}}}{\rho^{4} c_{2}^{2}}\right]^{g-3}+\ldots\right]
\end{gathered}
$$

As the factor associated with the $\mathrm{jth}$ term has a dependence on $\mathrm{j}$ given by $(4 e)^{j} e^{2 \sigma_{1}(2 j+1)} \sigma^{4 j^{2}}$, the series can be truncated essentially at $\mathrm{j}=\mathrm{O}(1)$, and a lower bound of the form $k_{1} k_{2}^{g}$ can be used for the sum in equation (8). As the integral over the multipliers is greater than $\frac{k_{1}}{\sqrt{2 \pi g}}\left(\frac{\pi k_{2} e^{1-2 \sigma_{1}} \rho^{4}}{2 c_{1}^{2}}\right)^{g} g^{g}$, it can be combined with the inequality for $[\operatorname{det}(\operatorname{Im} \tau)]^{-13}$ and the fixed point integral to give

$$
\begin{aligned}
\frac{k_{1} e^{-4 c_{2}}}{4} & \frac{\left|\xi_{11}^{0}-\xi_{g}^{0}\right|^{2}\left|\xi_{21}-\xi_{1 g}^{0}\right|^{2}}{\left|\xi_{11}^{0}-\xi_{21}^{0}\right|^{2}} \exp \left(\frac{-192 c^{4}}{c^{2}-2 c_{2}} \frac{d_{m a x}^{2}\left(\left\{I_{T_{n}}, I_{T_{n}}\right\}\right)}{d_{\min }^{2}\left(\left\{I_{T_{m}}\right\}\right)}\right) \\
& \left(\frac{1}{2}\right)^{1+\frac{192}{c_{2}} \frac{d_{\max }^{2}\left(\left\{I_{T_{n}}, I_{T_{n}^{-1}}\right\}\right)}{d_{\min }^{2}\left(\left\{I_{T_{m}}\right\}\right)}\left[c_{2} C_{0}+C_{1}-C_{0}\left(c^{2}+c-\frac{1}{2}\right)\right]} \\
& g^{-\frac{1}{2}}(2 \pi)^{16 g-3}\left(\frac{1}{\delta_{0}^{2}}-\frac{1}{\delta_{0}^{\prime 2}}\right)^{g-1}\left(\delta_{2}^{\prime 2}-\delta_{2}^{2}\right)^{g-2}\left(\frac{k_{2} e^{1-2 \sigma_{1}} \rho^{4}}{2 c_{1}^{2}}\right)^{g} \\
& \frac{g^{g}}{\left[\ln g+\frac{8 c}{c^{2}-2 c_{2}}-\ln c_{1}\right]^{13 g}}
\end{aligned}
$$

which is a refinement of the bound obtained in earlier work [9].

It has already been noted that the largest remainder terms in equation (6) would be of order $O\left(\frac{1}{\sqrt{g}}\right)$ for configurations of isometric circles of type (i). As these terms may not necessarily cancel at this order, an improved definition of the allowed ranges of values of the variables $\left|K_{n}\right|$ is required. In particular, upon using the formula for the period matrix 
elements, the inequalities $(\operatorname{Im} \tau)_{s s} \geq(\operatorname{Im} \tau)_{r r}, s \geq r$ only lead to constraints of the form $\frac{\left|K_{1}\right|}{\rho_{1}} \geq \frac{\left|K_{2}\right|}{\rho_{2}} \geq \ldots \geq \frac{\left|K_{g}\right|}{\rho_{g}}$ where $\rho_{n}=\prod_{\alpha}{ }^{(n, n)}\left|\frac{\xi_{1 n}-V_{\alpha} \xi_{2 n}}{\xi_{1 n}-V_{\alpha} \xi_{1 n}} \frac{\xi_{2 n}-V_{\alpha} \xi_{1 n}}{\xi_{2 n}-V_{\alpha} \xi_{2 n}}\right|$. Whereas integration of the absolute values $\left|K_{n}\right|$ over the range $\left[\frac{c_{1}}{g}, \frac{c_{2}}{g}\right]$ gives $\frac{1}{2^{g}}\left(\frac{1}{c_{1}^{2}}-\frac{1}{c_{2}^{2}}\right)^{g} g^{2 g}$, integration over the restricted range is bounded by $\frac{1}{2^{g} \rho_{1}^{2} \ldots \rho_{g}^{2} g !}\left(\frac{1}{\rho^{-2} c_{1}^{2}}-\frac{1}{\rho_{1}^{2} c_{2}^{2}}\right)^{g} g^{2 g}$. The modification associated with the dependence of the remainder terms in the product $\rho_{n}$ being $O\left(\frac{1}{\sqrt{g}}\right)$ does not affect the dominant behaviour of the bound, which is equivalent to that obtained in equation (10).

In the second type of configuration, all $2 \mathrm{~g}$ circles can be contained in a disk of finite radius of order $O\left(\frac{1}{\sqrt{g}}\right)$. The dominant contribution to the partition function is the fixed point integral

$$
\begin{aligned}
& \int \frac{1}{\operatorname{Vol}(S L(2, C))} \prod_{n=1}^{g} \frac{d^{2} \xi_{1 n} d^{2} \xi_{2 n}}{\left|\xi_{1 n}-\xi_{2 n}\right|^{4}} \\
& \geq \frac{\left|\xi_{11}^{0}-\xi_{1 g}^{0}\right|^{2}\left|\xi_{1 g}^{0}-\xi_{21}^{0}\right|^{2}}{\left|\xi_{11}^{0}-\xi_{21}^{0}\right|^{2}} \pi^{2 g-3} \delta_{2}^{\prime 2 g-4}\left[1-\frac{\left(\delta_{0}+\delta_{0}^{\prime}\right)^{2}}{4 \delta_{2}^{\prime 2}}\right]^{g-2} \\
& {\left[\frac{1}{\delta_{0}^{2}}\left[1+\frac{\epsilon_{0}^{\frac{1}{2}}}{1+\epsilon_{0}}\left[1-\sum_{n^{\prime}=1}^{\infty} \frac{\left(2 n^{\prime}-1\right) ! !}{\left(n^{\prime}+1\right) !} \frac{1}{2^{n^{\prime}}}\right]\right]^{-2}-\frac{1}{\delta_{0}^{\prime 2}}\right]^{g-1} g^{g-1} }
\end{aligned}
$$

where $\delta_{2}^{\prime}$ is the radius of the disk containing the isometric circles $\left\{I_{T_{n}}, I_{T_{n}^{-1}}, n=1, \ldots, g\right\}$. The conditions for the fundamental region of the symplectic modular group $\operatorname{Sp}(2 \mathrm{~g} ; \mathrm{Z})$ lead to a set of constraints $(\operatorname{Im} \tau)_{s s} \geq(\operatorname{Im} \tau)_{r r}, s \geq r$, which modify the integrals over the Schottky group parameters. The restrictions on the absolute values of the multipliers that might follow from such constraints are associated with a reduction by g!. As the magnitudes of the multiplier and fixed-point terms in $(\operatorname{Im} \tau)_{n n}$ are of the same order, the conditions on the period matrix lead to inequalities amongst a larger set of variables. It is shown in the appendix that a sequential ordering of $\left\{(\operatorname{Im} \tau)_{n n}\right\}$ can be obtained in a local neighbourhood consisting of $\mathrm{J}$ circles, with $\mathrm{J}$ bounded, by restricting the arguments of $\xi_{1 n}-\xi_{2 n}$ and $K_{n}$. The fixed-point integral will be reduced by an exponential function of the genus upon repeating the local ordering throughout the configuration. A global ordering can be achieved by choosing a generator associated with each neighbourhood $T_{r_{N}}$ 
and imposing constraints on either $\left|K_{r_{N}}\right|$ or $\left|\xi_{1 r_{N}}-\xi_{2 r_{N}}\right|$. The overall dependence of the combined integral over $\left\{K_{n}\right\}$ and $\left\{\xi_{1 n}, \xi_{2 n}\right\}$ will then be $k_{3}^{g} g^{g\left(1-\frac{1}{J}\right)}$.

The factorial growth is not changed substantially by other terms in the measure. Recall that

$$
\begin{aligned}
{[\operatorname{det}(\operatorname{Im} \tau)]^{-13}>(} & 2 \pi)^{13 g}\left[\ln \left(\frac{1}{\epsilon_{0}}\right)\right. \\
& \left.+\frac{1}{g} \sum_{n=1}^{g} \sum_{\alpha}{ }^{(n, n)} \frac{\left|\gamma_{\alpha}\right|^{-2}\left|\xi_{1 n}-\xi_{2 n}\right|^{2}}{\left|\xi_{1 n}+\frac{\delta_{\alpha}}{\gamma_{\alpha}}\right|\left|\xi_{2 n}+\frac{\delta_{\alpha}}{\gamma_{\alpha}}\right|\left|\xi_{1 n}-V_{\alpha} \xi_{1 n}\right|\left|\xi_{2 n}-V_{\alpha} \xi_{2 n}\right|}\right]^{-13 g}
\end{aligned}
$$

where $\sum_{\alpha}(n, n)$ excludes all elements $V_{\alpha}$ with left-most or right-most members equal to $T_{n}^{ \pm 1}$. In a hexagonal configuration, corresponding to the densest packing of the isometric circles, the circles are labelled by the level number $l$, which is related to the distance from the center of the domain enclosing all $I_{T_{n}}$ and $I_{T_{n}^{-1}}, n=1, \ldots, g$. The sum can be separated into a series involving the generators $T_{n_{l}}$ and a series associated with elements $V_{\alpha}$ that are products of two or more generators. A bound for the first series was obtained in an earlier investigation $[5]$.

It is shown in the appendix that the upper bound for the series involving elements $V_{\alpha}=T_{n_{l_{1}}}^{ \pm 1} \ldots T_{n_{l_{m}}}^{ \pm 1}$ is equal to $\frac{\delta_{0}^{\prime 2}}{\delta_{0}^{2}}\left[6 \frac{\delta_{0}^{\prime 2}}{\delta_{0}^{2}} \frac{\epsilon_{0}^{\prime}}{\left[1-\epsilon_{0}^{\prime}\right]^{2}}\right]^{m}$, multiplying mth order sums over fractions consisting of terms with leading-order behaviour $\left[l^{T_{n_{l_{1}}}^{ \pm 1}}\right]^{r_{1}} \ldots\left[l^{T_{n_{l_{m}}}^{ \pm 1}}\right]^{r_{m}}, \sum_{i=1}^{m} r_{i}=-(m+2)$, there entire sum is less than that of a convergent geometric series. An exponentially decreasing lower bound, therefore, is sufficient for $[\operatorname{det}(\operatorname{Im} \tau)]^{-13}$.

An evaluation of the primitive-element product factors requires an estimate of $\sum_{\alpha}{ }^{\prime}\left|K_{\alpha}\right|$ since

$$
\begin{aligned}
& \prod_{\alpha}{ }^{\prime}\left|1-K_{\alpha}\right|^{-1}>\exp \left(-\sum_{\alpha}{ }^{\prime}\left|K_{\alpha}\right|\right) \\
& \prod_{\alpha}^{\prime} \prod_{p=1}^{\infty}\left|1-K_{\alpha}^{p}\right|^{-1}>\exp \left(-\frac{1}{1-\epsilon_{0}^{\prime}} \sum_{\alpha}{ }^{\prime}\left|K_{\alpha}\right|\right)
\end{aligned}
$$

A genus-dependent bound can be obtained for the sum

$$
\sum_{\alpha}{ }^{\prime}\left|K_{\alpha}\right|=\sum_{\alpha}{ }^{\prime} \frac{\left|\gamma_{\alpha}\right|^{-2}}{\left|\xi_{1 \alpha}+\frac{\delta_{\alpha}}{\gamma_{\alpha}}\right|^{2}}<2 \frac{\epsilon_{0}^{\prime}}{\left(1-\epsilon_{0}^{\prime}\right)^{2}} \frac{\delta_{0}^{\prime 2}}{\delta_{0}^{2}} g+\sum_{V_{\tilde{\alpha}}=T_{n_{l}} V_{\beta}} \frac{\left|\gamma_{\tilde{\alpha}}\right|^{-2}}{\left|\xi_{1 \tilde{\alpha}}+\frac{\delta_{\tilde{\alpha}}}{\gamma_{\tilde{\alpha}}}\right|^{2}}
$$


which is demonstrated in the appendix to be only linearly increasing with respect to the genus as $g \rightarrow \infty$. Therefore, the primitive-element products are bounded below by an exponentially decreasing function of the genus, and the lower bound for the integral for configurations of isometric circles in category (ii) will be reduced further by an exponential factor.

The third category of isometric circles is defined by the parameter $\mathrm{q}, 0<q<\frac{1}{2}$, measuring the fall-off of the distances between the isometric circles. The multipliers and the distances between the fixed points are genus-dependent for these configurations. The Poincare series is bounded by

$$
\begin{aligned}
\sum_{\alpha}\left|\gamma_{\alpha}\right|^{-2}<2 \epsilon_{0}^{\prime} \mid & 1-\left.\frac{\epsilon_{0}^{\prime}}{g^{1-2 q}}\right|^{-2} \delta_{0}^{\prime 2} \\
& +\epsilon_{0}^{\prime 2}\left|1-\frac{\epsilon_{0}^{\prime}}{g^{1-2 q}}\right|^{-4} \frac{\delta_{0}^{\prime 4}}{g^{2}} \sum_{V_{\tilde{\alpha}}=T_{n_{l_{1}}}^{ \pm 1} T_{n_{l_{2}}}^{ \pm 1}}\left|\frac{\delta_{T_{n_{l_{1}}}^{ \pm 1}}}{\gamma_{T_{n_{l_{1}}}^{ \pm 1}}}+\frac{\alpha_{T_{n_{l_{2}}}^{ \pm 1}}}{\gamma_{T_{n_{l_{2}}}^{ \pm 1}}}\right|^{-2} \\
& +\sum_{V_{\tilde{\alpha}}=T_{n_{l}} V_{\beta}}\left|\gamma_{\tilde{\alpha}}\right|^{-2}
\end{aligned}
$$

where $V_{\beta}$ consists of two elements at least. An upper bound for the sum over the elements $V_{\tilde{\alpha}}=T_{n_{l_{1}}}^{ \pm 1} T_{n_{l_{2}}}^{ \pm 1}$ is found in the appendix to increase linearly with the genus. This will also hold true for $\sum_{\alpha}{ }^{\prime}\left|K_{\alpha}\right|$, and thus, the primitive-element products will be bounded below by an exponentially decreasing function of the genus.

The integrals over the multipliers and fixed points depend on $\mathrm{q}$, when $0<q<\frac{1}{2}$. For isometric circles defined by the limits in category (iii),

$$
\int \prod_{n=1}^{g} \frac{d^{2} K_{n}}{\left|K_{n}\right|^{4}}\left|1-K_{n}\right|^{4} \geq \pi^{g}\left(1-\epsilon_{0}^{\prime}\right)^{4 g}\left(\frac{1}{\epsilon_{0}^{2}}-\frac{1}{\epsilon_{0}^{\prime 2}}\right)^{g} g^{2(1-2 q) g}
$$




$$
\begin{aligned}
& \int \frac{1}{\operatorname{Vol}(S L(2, \mathbb{C}))} \prod_{n=1}^{g} \frac{d^{2} \xi_{1 n} d^{2} \xi_{2 n}}{\left|\xi_{1 n}-\xi_{2 n}\right|^{4}} \\
& \geq \frac{\left|\xi_{11}^{0}-\xi_{1 g}^{0}\right|^{2}\left|\xi_{1 g}^{0}-\xi_{21}^{0}\right|^{2}}{\left|\xi_{11}^{0}-\xi_{21}^{0}\right|^{2}} \pi^{2 g-3} \delta_{2}^{\prime 2 g-4}\left[1-\frac{\left(\delta_{0}+\delta_{0}^{\prime}\right)^{2}}{4 \delta_{2}^{\prime 2} g^{1-2 q}}\right]^{g-2} \\
& {\left[\frac{1}{\delta_{0}^{2}}\left[1+\frac{\epsilon_{0}^{\frac{1}{2}}}{1+\epsilon_{0}}\left[1-\sum_{n^{\prime}=1}^{\infty} \frac{\left(2 n^{\prime}-1\right) ! !}{\left(n^{\prime}+1\right) !} \frac{1}{2^{n^{\prime}}}\right]\right]^{-2}-\frac{1}{\delta_{0}^{\prime 2}}\right]^{g-1} g^{2(g-1) q} }
\end{aligned}
$$

There is also a qualitative difference between the lower bound for $[\operatorname{det}(\operatorname{Im} \tau)]^{-13}$ when $q<\frac{1}{2}$ and the same bound for $q=\frac{1}{2}$.

$$
[\operatorname{det}(\operatorname{Im} \tau)]^{-13}>(2 \pi)^{13 g}\left[(1-2 q) \ln g-\ln \epsilon_{0}+\ldots\right]^{-13 g}
$$

Combining these inequalities gives a growth of $\frac{k^{g} g^{2(g-g q-q)}}{(\ln g)^{13 g}}$. Since the multiplier term in $\operatorname{Im} \tau$ grows more rapidly than the sum of the logarithms of the fixed-point ratios, the inequalities $\frac{\epsilon_{0}}{g^{1-2 q}} \leq\left|K_{g}\right| \leq \rho^{2}\left|K_{g-1}\right|, \ldots,\left|K_{1}\right| \leq \frac{\epsilon_{0}^{\prime}}{g^{1-2 q}}$ where $\rho$ was defined by equation (6). Division by a factor of $\rho^{-2 g} g$ ! would lead to an overall dependence on the genus equal to $(k \cdot e)^{g} \rho^{2 g} \frac{g^{(g-2 q g-2 q)}}{(\ln g)^{13 g}}$. The total contribution of the configurations in category (iii) can be determined by an overlapping of the intervals for allowed values of $\left|K_{n}\right|, \mathrm{n}=1, \ldots, \mathrm{g}$, corresponding to different q. Selecting a specific value of the genus $g_{0}$, the upper limit of one interval can be set equal to the lower limit of the next interval. Therefore, $\frac{\epsilon_{0}}{g_{0}^{1-2 q_{1}}}=\frac{\epsilon_{0}^{\prime}}{g_{0}}$, implying that $\epsilon_{0}=\epsilon_{0}^{\prime} g_{0}^{-2 q_{1}}$, and $\frac{\epsilon_{0}}{g_{0}^{1-2 q_{n}}}=\frac{\epsilon_{0}^{\prime}}{g_{0}^{1-2 q_{n-1}}}$, so that $q_{n}=n q_{1}$. There exists an integer $N=\frac{1}{2 q_{1}}+r\left(q_{1}\right), 0 \leq r\left(q_{1}\right)<1$ such that $q_{N} \leq \frac{1}{2}$ and $q_{N+1} \geq \frac{1}{2}$. As $q_{1}=\frac{\ln \left(\frac{\epsilon_{0}^{\prime}}{\epsilon_{0}}\right)}{2 \ln g_{0}}$, for arbitrarily large genus $g$, the integer $\frac{\ln g}{\ln \left(\frac{\epsilon_{0}^{\prime}}{\epsilon_{0}}\right)}+r\left(\epsilon_{0}, \epsilon_{0}^{\prime}, g\right)$ equals the number of discrete evenly spaced values of $q$ in the interval $\left(0, \frac{1}{2}\right)$ selected by the requirement of non-overlapping of the ranges for $\left|K_{n}\right|$. The sum of the contributions to the partition function integral associated with the conditions $\frac{\epsilon_{0}}{g^{1-2 q_{i}}}<\left|K_{n}\right|<\frac{\epsilon_{0}^{\prime}}{g^{1-2 q_{i}}}$, and $\frac{\delta_{0}}{g^{q_{i}}}<\left|\xi_{1 n}-\xi_{2 n}\right|<\frac{\delta_{0}^{\prime}}{g^{q_{i}}}, \mathrm{i}=1, \ldots, \mathrm{N}$ is

$$
\frac{(k \cdot e)^{g} \rho^{2 g} g^{g}}{(\ln g)^{13 g}} e^{-2 \ln \left(\frac{\epsilon_{0}^{\prime}}{\epsilon_{0}}\right)(g+1)}\left[\frac{1-g^{-2(g+1)}}{1-e^{-2 \ln \left(\frac{\epsilon_{0}^{\prime}}{\epsilon_{0}}\right)(g+1)}}\right]
$$


The potential infinity associated with the continuous parameter q does not occur.

However, each value of $\mathrm{q}$ defines a different genus-dependence of the variables $\left|K_{n}\right|$ and $\left|\xi_{1 n}-\xi_{2 n}\right|$. Magnitudes of the multipliers may be grouped according to the value of q and inequalities can be imposed within each group. As the ranges of $\left|K_{n}\right|$ do not overlap for different choices of $\mathrm{q}$, the inequalities $(\operatorname{Im} \tau)_{s s} \geq(\operatorname{Im} \tau)_{r r}$ will be satisfied for all $s \geq r$. Integration of the multipliers in each group can be performed subject to the constraints on the absolute values, and the integrals will be equal to those given previously, with a reduced number of variables. The number of groupings is the number of ways of partitioning g ordered objects into $\frac{\ln g}{\ln \left(\frac{\epsilon_{0}^{\prime}}{\epsilon_{0}}\right)}+r\left(\epsilon_{0}, \epsilon_{0}^{\prime}, g\right)$ different sets. This introduces a combinatorial factor

$$
\frac{\left(g+\frac{\ln g}{\ln \left(\frac{\frac{\epsilon}{0}_{0}^{\prime}}{\epsilon_{0}}\right)}-1\right) !}{g !\left(\frac{\ln g}{\ln \left(\frac{\epsilon_{0}^{\prime}}{\epsilon_{0}}\right)}-1\right) !}
$$

which can be used to estimate the increase in the lower bound for the regularized string partition function.

\section{A Separate Category of Isometric Circles}

There are other configurations of isometric circles that are consistent with the genusindependent cut-off imposed on the length of closed geodesics rendering the moduli space integral finite. A disk of radius $O(\sqrt{g})$ containing circles of radius $\mathrm{O}(1)$ can be projected onto a sphere with handles of thickness $O\left(\frac{1}{\sqrt{g}}\right)$. As a conformal transformation can be chosen to change the metric to one of constant negative curvature, so that for surfaces in the $R=-1$ slice of Teichmuller space, the area increases linearly with the genus and the thickness of the handles will be expanded by a factor of $\sqrt{g}$ to $\mathrm{O}(1)$ in the intrinsic metric.

Each of the terms in the measure for the integral (2) could be evaluated for this configuration. However, $\left|\gamma_{\alpha}\right|^{-2}$ is of order $\mathrm{O}(1)$ when $V_{\alpha}=T_{n_{1}} T_{n_{2}}$, because the spacing between $I_{T_{n_{1}}}$ and $I_{T_{n_{2}}}^{-1}$ is of order $\mathrm{O}(1)$ for neighbouring circles. The Poincare series $\sum_{\alpha}\left|\gamma_{\alpha}\right|^{-2}$ 
would diverge even at finite genus. Bounds for the primitive-element products may exist as the arguments of the multipliers tend to cancel. The determinant factor $[\operatorname{det}(\operatorname{Im} \tau)]^{-13}$ also may be bounded below. It can be seen that the contribution of this configuration to the partition function is not significant, without requiring an evaluation of these bounds, because the integrals over the multipliers and fixed points only increase exponentially as $\left|K_{n}\right|=O(1)$ and $\left|\xi_{1 n}-\xi_{2 n}\right|=O(1)$.

Configurations of isometric circles involving fixed-point distances of $O\left(g^{r}\right), r>0$, also do not contribute significantly to the partition function [5].

\section{Upper Bounds}

The setting of upper bounds for the integrals over domains in moduli space is required for a full analysis of the summability of the perturbation series. Considering the intermediate configurations in category (iii), with $0<q<\frac{1}{2}$, one finds

$$
\begin{gathered}
\int \prod_{n=1}^{g} \frac{d^{2} K_{n}}{\left|K_{n}\right|^{4}}\left|1-K_{n}\right|^{4} \leq \pi^{g}\left(1-\epsilon_{0}\right)^{4 g}\left(\frac{1}{\epsilon_{0}^{2}}-\frac{1}{\epsilon_{0}^{\prime 2}}\right)^{g} g^{(1-2 q) g} \\
\int \frac{1}{\operatorname{Vol}(S L(2, \mathbb{C}))} \prod_{n=1}^{g} \frac{d^{2} \xi_{1 n} d^{2} \xi_{2 n}}{\left|\xi_{1 n}-\xi_{2 n}\right|^{4}} \leq \frac{\left|\xi_{11}^{0}-\xi_{1 g}^{0}\right|^{2}\left|\xi_{1 g}^{0}-\xi_{21}^{0}\right|^{2}}{\left|\xi_{11}^{0}-\xi_{21}^{0}\right|^{2}} \pi^{2 g-3} \\
\cdot \delta_{2}^{2 g-4}\left[\frac{1}{\delta_{0}^{2}}-\frac{1}{\delta_{0}^{\prime 2}}\right]^{g-1} g^{2(g-1) q}
\end{gathered}
$$

The logarithmic increase of the diagonal entries of $\operatorname{Im} \tau$ suggests that $\operatorname{det}(\operatorname{Im} \tau)$ is a rapidly increasing function of the genus. The determinant is equal to the volume of the parallelepiped spanned by the basis vectors $\underline{v}_{1}, \ldots, \underline{v}_{g}$ representing the rows of the matrix. Denoting $\theta_{1 \ldots n}$ to be the angle from $\underline{v}_{n}$ to the hyperplane spanned by $\underline{v}_{1}, \ldots, \underline{v}_{n-1}$ and since $\sin \theta_{1 \ldots n}=O(1)$ when the off-diagonal entries are $\mathrm{O}(1)$, it follows that $\operatorname{det}(\operatorname{Im} \tau) \geq$ $d(q)^{g} O(\ln g)^{g}$ and

$$
[\operatorname{det}(\operatorname{Im} \tau)]^{-13}<(d(q))^{-13 g}(2 \pi)^{13 g}(\ln g+O(1))^{-13 g}
$$

The primitive-element product can be bounded above because

$\prod_{\alpha}{ }^{\prime}\left|1-K_{\alpha}\right|^{-1}<\exp \left(\sum_{\alpha}{ }^{\prime}\left|K_{\alpha}\right|\right)$, and for each of the configurations, this function is an 
exponentially increasing function of the genus. It follows that the precise growth of the regularized integral, is equivalent, up to an exponential factor, for the upper and lower bounds. The increase of factorial type with respect to the genus remains unaltered.

\section{Graph Theory Combinatorics}

The upper and lower bounds for the partition function have been found by using the measure defined in terms of multipliers $K_{n}$ and fixed points $\xi_{1 n}$ and $\xi_{2 n}$ in the uniformization of Riemann surfaces by Schottky groups. The Schottky covering is intermediate between the Riemann surface and the simply connected covering obtained by cutting both the a- and b-cycles, which are the $2 \mathrm{~g}$ independent homologically non-trivial paths along the g handles. Every compact surface of genus $g \geq 2$ can be represented as the quotient $\mathrm{H} / \mathrm{G}$, where $\mathrm{H}$ is the upper half plane and $\mathrm{G}$ is a discrete subgroup of $\operatorname{PSL}(2, \mathbb{R})$ consisting of hyperbolic elements. The elements of $\mathrm{G}$ are conjugated to $\left(\begin{array}{cc}e^{\frac{l}{2}} & 0 \\ 0 & e^{-\frac{l}{2}}\end{array}\right)$, where $l$ can be interpreted as the length of a corresponding closed geodesic on the Riemann surface [10]. Primitive elements of the group G, which are not powers of the other elements, correspond to simple closed geodesics. Denoting the primitive elements by the index set $\{\gamma\}$, the length spectrum $\left\{l_{\gamma}\right\}$ always has a minimum value $l_{0}$ for compact surfaces. The Selberg trace function is defined by

$$
Z(s)=\prod_{\gamma}{ }^{\prime} \prod_{n=0}^{\infty}\left[1-e^{-(s+n) l_{\gamma}}\right]
$$

The partition function is $\sum_{g=0}^{\infty} \kappa^{g} \int_{M_{g}} d \mu_{W P} Z(2) Z^{\prime}(1)^{-13}$ where $M_{g}$ is the moduli space of genus g surfaces and $d \mu_{W P}$ is the Weil-Petersson measure. If the minimum length $l_{0}$ is bounded below by $\bar{l}_{0}$ in $\bar{M}_{g}-N\left(D_{g}\right)$ where $\bar{M}_{g}$ is the closure of $M_{g}$ and $N\left(D_{g}\right)$ is a neighbourhood of the compactification divisor, it can be shown that $Z(2) Z^{\prime}(1)^{-13}>c_{1}\left(\bar{l}_{0}\right) c_{2}\left(\bar{l}_{0}\right)^{g}$. The integral over the Weil-Petersson measure in a region in Teichmuller space can be estimated. Any Riemann surface of finite genus can be viewed as a thickened trivalent graph with branches at the final level intertwined. The number of different pairings of the $2 \mathrm{~g}$ branches is $(2 \mathrm{~g}-1) ! !$. This is consistent with the counting of non-isomorphic trivalent graphs [11]. 
The number of cells may increase at a factorial rate, but it remains to be shown that the cells are non-intersecting and that they belong to a single fundamental region of the symplectic group. This requires a study of the transformation between surfaces formed out of graphs with different branches intertwined. If the transformation does not lie in the identity component of the diffeomorphism group, then the surfaces lie in different fundamental domains of the mapping class group, and both cannot be included in the counting. Integration over the length and twist parameters suggests that the volume occupied by each cell depends exponentially on the genus, so that the growth will be determined by the restriction to the fundamental domain of the modular group.

In this connection, it may be observed that a trivalent graph in a plane will have intersecting branches at large order if the length of the branches is fixed at a constant value. If the graph is then thickened to obtain a surface in $\mathbb{R}^{3}$ with minimal genusindependent cross-sectional area, the disks at the ends of the branches at the final level will lie in overlapping cylindrical regions, and it will not be possible to join the pairs of disks without joining other disks. This property, which would continue to hold in $\mathbb{R}^{26}$, suggests that the counting of closed surfaces at finite genus would changed by the overlapping of the regions. Intersection of the branches may be avoided if the lengths of the branches are allowed to increase exponentially with respect to the order. An exponential increase would reduce the integration over the length parameters of all of the $\mathrm{g}$ handles by a factor which would affect the factorial growth obtained by counting of the intertwined graphs. An exponential decrease of the length of the branches would also eliminate intersections, but it is not consistent with a minimal genus-independent cross-sectional area.

Thus, the factorial growth derived from graph theory appears to be linked to the emergence of a point-like structure associated with the boundaries of the string worldsheet in the infinite-genus limit. If the constraint $\left|\gamma_{n}\right|^{-2} \sim \frac{1}{g}$ is not imposed on the Schottky group parameters, then a factorial increase can be easily obtained through integration of the variables $\left|K_{n}\right|$ or $\left|\xi_{1 n}-\xi_{2 n}\right|$ [6]. The surfaces giving rise to these divergences in this limit, having boundaries of zero linear measure, are eliminated by the genus-independent cut-off on the lengths of the closed geodesics. The reduction in the growth of the string integral 
has been verified in the calculations of the bounds in Sections 2 and 4, using the Schottky group parametrization.

\section{The Partition Function for a Curved Background}

The partition function for a string propagating in a curved background would be

$$
Z=\sum_{g=0}^{\infty} \kappa^{g} \int \frac{D\left[h_{\alpha \beta}\right] D\left[X^{\mu}\right]}{\operatorname{Vol}\left(\operatorname{Diff} \Sigma_{g}\right) \operatorname{Vol}\left(\operatorname{Conf} \Sigma_{g}\right)} e^{-\frac{1}{2} \int d^{2} \xi \sqrt{h} h^{\alpha \beta} \partial_{\alpha} X^{\mu} \partial_{\beta} X^{\nu} g_{\mu \nu}}
$$

Formulas for partition functions of closed bosonic strings have been obtained for conformally flat backgrounds [12] and group manifolds [13].

Divergences in the partition function may be identified with particular factors in the measure. In flat space, the general bosonic N-string g-loop amplitude can be obtained by sewing together g pairs of legs of an $(\mathrm{N}+2 \mathrm{~g})$-string tree amplitude. The integrand of the N-string amplitude can be written as

$$
d^{N-3} W_{N}(X, b, c)=\int d^{N} \hat{c} e^{-S(X, b, c+\hat{c})}(2 \pi)^{26} \delta\left(\sum_{i=1}^{N} p_{i}\right)
$$

where $p_{i}$ are the string momenta, $\mathrm{S}(\mathrm{X}, \mathrm{b}, \mathrm{c})$ is the string action involving coordinate and ghost fields, and the ghost modes $\hat{c}$ are needed to reproduce Koba-Nielsen-type variables. An M-string vertex can be sewed with an N-string vertex along one of the legs to give an $(\mathrm{M}+\mathrm{N}-2)$-string-tree amplitude with integrand

$$
\begin{aligned}
d^{(M+N-5)} W_{M+N-2}\left(X+T X^{\prime}, b+T b^{\prime}, c+T c^{\prime}\right) & \\
=(2 \pi)^{26} \int & D\left[X_{s}, b_{s}, c_{s}\right] d c_{s} \delta^{26}\left(p_{s}\right) e^{-S\left[x_{s}, b_{s}, c_{s}+\hat{c_{s}}\right]} \\
& d^{(M-3)} W_{M}(X, b, c) d^{(N-3)} W_{N}\left(X^{\prime}, b^{\prime}, c^{\prime}\right)
\end{aligned}
$$

with $X_{s}, b_{s}, c_{s}$ and $\hat{c_{s}}$ being sewing fields and $\mathrm{T}$ being the projective transformation connecting the two legs [8]. Each sewing of a pair of legs will introduce one modular integration variable through the sewing field $c_{s}$. Sewing g pairs of legs of a 2 g-string tree amplitude will involve 3g-3 integration variables for moduli space. The projective transformations for $g$ sewings generate the Schottky group uniformizing the Riemann surface. Several of 
the factors in the measure can be traced to integration of the sewing fields. The string sewing fields $\left\{X_{s n}\right\}$ give the factors $(\operatorname{det} \operatorname{Im} \tau)^{-13} \prod_{\alpha}{ }^{\prime} \prod_{p=1}^{\infty}\left|1-K_{\alpha}^{p}\right|^{-52}$, while the ghost sewing fields $\left\{b_{s n}, c_{s n}\right\}$ give $\prod_{\alpha}{ }^{\prime} \prod_{p=1}^{\infty}\left|1-K_{\alpha}\right|^{4}$. The remaining factors may be obtained through integration over the ghost zero modes.

It has been shown that divergences in the regularized partition function result from factors associated with the ghost zero modes, although the logarithmic factor, which could affect summability of the perturbation series, arises from $(\operatorname{det} \operatorname{Im} \tau)^{-13}$. In curved space, a similar derivation of the factor in the measure might be feasible, with the path integration being modified by the change in the metric.

Without explicitly deriving the measure, it can be seen that the finite volume of a compact target space would lead to a cut-off in the volume of the string world-sheet if the mapping between the target-space metric and the intrinsic world-sheet metric is differentiable and invertible. This would result in the exclusion of certain configurations of isometric circles in the large-genus limit. It has been demonstrated here that the classification of these configurations is related to the type of divergence in the perturbation series. Thus, the inclusion of only particular configurations of isometric circles for a world-sheet embedded in a compact curved target manifold would alter the Borel summability of the series.

\section{Acknowledgements}

This research was initiated at the I.C.T.P., Trieste, where financial support was received from the Atomic Energy Commission, Vienna. 


\section{Appendix}

For the second category of isometric circles, $\left|\xi_{1 n}-\xi_{2 n}\right|=O\left(\frac{1}{\sqrt{g}}\right)$, so that the dominant contribution to the fixed-point sum in $\operatorname{Im} \tau$ arises from the group of neighbouring isometric

circles $I_{V_{\alpha}}$ such that $\left|\xi_{1 s}+\frac{\delta_{\alpha}}{\gamma_{\alpha}}\right|=O\left(\frac{1}{\sqrt{g}}\right)$ and $\left|\xi_{1 s}-V_{\alpha} \xi_{1 s}\right|=O\left(\frac{1}{\sqrt{g}}\right)$. The fixed-point term in $\operatorname{Im} \tau$ will then be $\mathrm{O}(1)$, which is the same order of magnitude as $\ln \left(\frac{1}{\left|K_{n}\right|}\right)$. Thus, inequalities amongst the fixed-point variables and even the arguments of the multipliers may be used as alternative representations of the conditions $(\operatorname{Im} \tau)_{s s} \geq(\operatorname{Im} \tau)_{r r}, s \geq r$.

Moreover, the radii $\left|\gamma_{\alpha}\right|^{-1}$ decrease as the number of generators in $V_{\alpha}$ increases, and an even distribution of the phases of $\gamma_{\alpha}^{-2}$ in the interval $[-\pi, \pi]$ leads to a cancellation of terms in the sum. It will be sufficient, therefore, to consider the isometric circles $I_{T_{n_{l}}^{(j)}}$ of the fundamental generators, where $l$ is the level number in the hexagonal configuration and $(\mathrm{j})$ labels the $6 \mathrm{l}-6$ circles at each level. Let

$$
\begin{aligned}
C_{l, s}^{(j)} & =\frac{\left|\xi_{1 s}-\xi_{2 s}\right|^{2}}{\left|\xi_{1 s}+\frac{\delta_{T_{n}^{(j)}}}{\gamma_{T_{n_{l}}(j)}}\right|\left|\xi_{2 s}+\frac{\delta_{T_{n_{l}}^{(j)}}}{\gamma_{T_{n_{l}}^{(j)}}}\right| \xi_{1 s}-T_{n_{l}}^{(j)} \xi_{1 s}|| \xi_{2 s}-T_{n_{l}}^{(j)} \xi_{2 s} \mid} \\
D_{l, s}^{(j)} & =\left|\gamma_{n_{l}}^{(j)}\right|^{-2} C_{l, s}^{(j)} \cos \left[\arg \left(\frac{K_{n_{l}}^{(j)}\left(\xi_{2 s}-T_{n_{l}}^{(j)} \xi_{2 s}\right)^{-1}}{\left(1-K_{n_{l}}^{(j)}\right)^{2}\left(\xi_{1 s}+\frac{\delta_{n_{l}}^{(j)}}{\gamma_{n_{l}}^{(j)}}\right)\left(\xi_{2 s}+\frac{\delta_{n_{l}}^{(j)}}{\gamma_{n_{l}}^{(j)}}\right)\left(\xi_{1 s}-T_{n_{l}}^{(j)} \xi_{1 s}\right)}\right)\right] \\
E_{l, s}^{(j)} & =\sqrt{\left|\gamma_{n_{l}}^{(j)}\right|^{-4} C_{l, s}^{(j) 2}-D_{l, s}^{(j) 2}}
\end{aligned}
$$

Then, if two pairs of fixed points $\left(\xi_{1 r}, \xi_{2 r}\right)$ and $\left(\xi_{1 s}, \xi_{2 s}\right)$ are located near to each other, and if the difference in the arguments of $\left(\xi_{1 r}-\xi_{2 r}\right)$ and $\left(\xi_{1 s}-\xi_{2 s}\right)$ is denoted by $\epsilon_{r s}$, the inequalities $(\operatorname{Im} \tau)_{s s} \geq(\operatorname{Im} \tau)_{r r}, s \geq r$ will be satisfied when

$$
\begin{aligned}
\epsilon_{r s} \geq \frac{1}{2} \arcsin & {\left[\frac { 1 } { 2 } \left[\sum _ { l = 2 } ^ { J } \sum _ { \substack { j = 1 \\
[ l ( j ) ] \neq r , s ] } } ^ { 6 l - 6 } \left[D_{l, s}^{(j)} \sin \left[2 \arg \left(\xi_{1 l}^{(j)}-\xi_{2 l}^{(j)}\right)+2 \arg \left(\xi_{1 s}-\xi_{2 s}\right)\right]\right.\right.\right.} \\
& \left.\left.\left.+E_{l, s}^{(j)} \cos \left[2 \arg \left(\xi_{1 l}^{(j)}-\xi_{2 l}^{(j)}\right)+2 \arg \left(\xi_{1 s}-\xi_{2 s}\right)\right]\right]\right]^{-1} \cdot \ln \left(\frac{\epsilon_{0}^{\prime}}{\epsilon_{0}}\right)\right]
\end{aligned}
$$

where $\mathrm{J}$ is bounded, because the dominant contribution to the fixed point sum corresponds 
to the isometric circles $I_{T_{n_{l}}^{(j)}}$ and $I_{T_{n_{l}}^{(j)}}^{-1}$ near to the circles $I_{T_{s}}$ and $I_{T_{s}-1}$. Since $\left|\gamma_{n_{l}}^{(j)}\right|^{-2}=$ $O\left(\frac{1}{g}\right), C_{l, s}^{(j)}=O(g), D_{(l, s)}^{(j)}=O(1)$ and $E_{l, s}^{(j)}=O(1), \epsilon_{r s}=O(1)$.

It may be assumed that the range of arguments and absolute values of $K_{n_{l}}^{(j)}$ can be selected to be sufficiently narrow so that it will not affect the inequalities for the fixedpoint ratios significantly. Given a range of values for $\arg \left(\xi_{1 s}-\xi_{2 s}\right)$ and a choice for $\arg \left(\xi_{1 l}^{(j)}-\xi_{2 l}^{(j)}\right)$, the shifted range of values for $\arg \left(\xi_{1 r}-\xi_{2 r}\right)$ can be obtained. Therefore, the ranges of the arguments $\arg \left(\xi_{1 r_{n}}-\xi_{2 r_{n}}\right)$ can be ordered sequentially. An alternative method, which allows $\arg \left(\xi_{1 l}^{(j)}-\xi_{2 l}^{(j)}\right)$ to be arbitrary initially, would involve a coupled set of equations resulting from inequalities of the type given in equation (A.2). As a consequence of the width of the angular intervals being of order $\mathrm{O}(1)$, the fixed point integral is reduced by an exponential factor.

The bound for the determinant factor $[\operatorname{det}(\operatorname{Im} \tau)]^{-13}$ has been given in Section 2 . The fixed-point sum in the lower bound can be shown to be finite for arbitrary genus [5]. The sum can be expressed as

$$
\begin{aligned}
& \sum_{n_{l} \neq n} \frac{\left|\gamma_{n_{l}}\right|^{-2}\left|\xi_{1 n}-\xi_{2 n}\right|^{2}}{\left|\xi_{1 n}-T_{n_{l}} \xi_{1 n}\right|\left|\xi_{2 n}-T_{n_{l}} \xi_{2 n}\right|\left|\xi_{1 n}+\frac{\delta_{n_{l}}}{\gamma_{n_{l}}}\right|\left|\xi_{2 n}+\frac{\delta_{n_{l}}}{\gamma_{n_{l}}}\right|} \\
& \quad+\sum_{n_{l} \neq n} \frac{\left|\gamma_{n_{l}}\right|^{-2}\left|\xi_{1 n}-\xi_{2 n}\right|^{2}}{\left|\xi_{1 n}-T_{n_{l}}^{-1} \xi_{1 n}\right|\left|\xi_{2 n}-T_{n_{l}}^{-1} \xi_{2 n}\right|\left|\xi_{1 n}-\frac{\alpha_{n_{l}}}{\gamma_{n_{l}}}\right|\left|\xi_{2 n}-\frac{\alpha_{n_{l}}}{\gamma_{n_{l}}}\right|} \\
& \quad+\sum_{V_{\tilde{\alpha}}=T_{n_{l}} V_{\beta}}^{(n, n)} \frac{\left|\gamma_{\tilde{\alpha}}\right|^{-2}\left|\xi_{1 n}-\xi_{2 n}\right|^{2}}{\left|\xi_{1 n}-V_{\tilde{\alpha}} \xi_{1 n}\right|\left|\xi_{2 n}-V_{\tilde{\alpha}} \xi_{2 n}\right|\left|\xi_{1 n}+\frac{\delta_{\tilde{\alpha}}}{\gamma_{\tilde{\alpha}}}\right|\left|\xi_{2 n}+\frac{\delta_{\tilde{\alpha}}}{\gamma_{\tilde{\alpha}}}\right|}
\end{aligned}
$$

Suppose that $\xi_{1 n}$ and $\xi_{2 n}$ lie at the center of a hexagonal arrangement of $2 \mathrm{~g}$ isometric circles spaced apart from each other by a distance of $O\left(\frac{1}{\sqrt{g}}\right)$. Since

$$
\begin{aligned}
& \left|\xi_{2 n}+\frac{\delta_{n_{l}}}{\gamma_{n_{l}}}\right| \geq(l-1) \frac{\delta_{0}}{\sqrt{g}}\left|\xi_{1 n}+\frac{\delta_{n_{l}}}{\gamma_{n_{l}}}\right| \geq \max \left\{\left[l-1-\frac{\delta_{0}^{\prime}}{\delta_{0}}\right] \frac{\delta_{0}}{\sqrt{g}}, \frac{\delta_{0}}{\sqrt{g}}\right\} \\
& \left|\xi_{2 n}-T_{n_{l}} \xi_{2 n}\right| \geq \max \left\{\left[l-1-\frac{\delta_{0}^{\prime}}{\delta_{0}}\left[1+\frac{2 \epsilon_{0}^{\prime \frac{1}{2}}}{1-\epsilon_{0}^{\prime}}\right]\right] \frac{\delta_{0}}{\sqrt{g}}, \frac{\delta_{0}}{\sqrt{g}}\right\} \\
& \left|\xi_{1 n}-T_{n_{l}} \xi_{1 n}\right| \geq \max \left\{\left[l-1-2 \frac{\delta_{0}^{\prime}}{\delta_{0}}\left[1+\frac{\epsilon_{0}^{\prime \frac{1}{2}}}{1-\epsilon_{0}^{\prime}}\right]\right] \frac{\delta_{0}}{\sqrt{g}}, \frac{\delta_{0}}{\sqrt{g}}\right\}
\end{aligned}
$$


and as there are only $6(1-1)$ circles at level 1 in a configuration with $\left[\frac{1}{2}+\frac{1}{6} \sqrt{9+24 g}\right]$ levels, the first two sums in (A.3) converge as $g \rightarrow \infty$.

For the sum over elements $V_{\tilde{\alpha}}=T_{n_{l}} V_{\beta}$, the ratio of the square of the radii of $I_{V_{\tilde{\alpha}}}$ and $I_{V_{\beta}}$ is

$$
\left|\frac{\gamma_{\tilde{\alpha}}}{\gamma_{\beta}}\right|^{-2}=\left|\gamma_{n_{l}}\right|^{-2}\left|\frac{\delta_{n_{l}}}{\gamma_{n_{l}}}+\frac{\alpha_{\beta}}{\gamma_{\beta}}\right|^{-2}<\frac{\epsilon_{0}^{\prime}}{\left[1-\epsilon_{0}^{\prime}\right]^{2}} \frac{\delta_{0}^{\prime 2}}{g}\left|\frac{\delta_{n_{l}}}{\gamma_{n_{l}}}+\frac{\alpha_{\beta}}{\gamma_{\beta}}\right|^{-2}
$$

It follows that the coefficients multiplying the convergent jth order sums of fractions involving the level numbers are $\frac{\delta_{0}^{\prime 2}}{\delta_{0}^{2}}\left[6 \frac{\delta_{0}^{\prime 2}}{\delta_{0}^{2}} \frac{\epsilon_{0}^{\prime}}{\left(1-\epsilon_{0}^{\prime}\right)^{2}}\right]^{j}$, and the entire fixed point sum containing $\xi_{1 n}$ and $\xi_{2 n}$ is bounded by a convergent geometric series if $6 \frac{\delta_{0}^{\prime 2}}{\delta_{0}^{2}} \frac{\epsilon_{0}^{\prime}}{\left[1-\epsilon_{0}^{\prime}\right]^{2}}<\Delta$, where $\Delta$ is a constant determined by the level number sums.

A similar method can be used for estimating the sum $\sum_{\alpha}{ }^{\prime}\left|K_{\alpha}\right|$. The first set of elements included in the sum over $V_{\tilde{\alpha}}=T_{n_{l}} V_{\beta}$ in equation (14) is of the form $\left\{T_{n_{l_{1}}} T_{n_{l_{2}}}\right\}$. For these elements $\xi_{1 \tilde{\alpha}} \in D_{V_{\tilde{\alpha}}^{-1}} \subset D_{T_{n_{l_{1}}}}^{-1}$ and $-\frac{\delta_{\tilde{\alpha}}}{\gamma_{\tilde{\alpha}}} \in D_{V_{\tilde{\alpha}}} \subset D_{T_{n_{l_{2}}}}$. Denoting the level of $I_{T_{n_{l_{1}}}}$ to be $l$ and the level of $I_{T_{n_{l_{2}}}^{-1}}$ to be $l+l_{0}$, it follows that $\left|\xi_{1 \tilde{\alpha}}+\frac{\delta_{\tilde{\alpha}}}{\gamma_{\tilde{\alpha}}}\right| \geq \frac{\delta_{0}}{\sqrt{g}}$ when

$$
\begin{aligned}
& \left|l_{0}\right| \leq\left\{2 \frac{\delta_{0}^{\prime}}{\delta_{0}}\left(1+\frac{2 \epsilon_{0}^{\prime \frac{1}{2}}}{1-\epsilon_{0}^{\prime}}\right)\right\} \text { and }\left|\xi_{1 \tilde{\alpha}}+\frac{\delta_{\tilde{\alpha}}}{\gamma_{\tilde{\alpha}}}\right| \geq\left[\left|l_{0}\right|-2 \frac{\delta_{0}^{\prime}}{\delta_{0}}\left(1+\frac{2 \epsilon_{0}^{\prime \frac{1}{2}}}{1-\epsilon_{0}^{\prime}}\right)\right] \frac{\delta_{0}}{\sqrt{g} \text { when }} \\
& \left|l_{0}\right| \geq\left\{2 \frac{\delta_{0}^{\prime}}{\delta_{0}}\left(1+\frac{2 \epsilon_{0}^{\prime \frac{1}{2}}}{1-\epsilon_{0}^{\prime}}\right)\right\}+1 \text {. Then }
\end{aligned}
$$

$$
\left|\gamma_{\tilde{\alpha}}\right|^{-2}=\left|\gamma_{n_{l_{1}}}\right|^{-2}\left|\gamma_{n_{l_{2}}}\right|^{-2}\left|\frac{\delta_{n_{l_{1}}}}{\gamma_{n_{l_{1}}}}+\frac{\alpha_{n_{l_{2}}}}{\gamma_{n_{l_{2}}}}\right|^{-2} \leq \frac{\epsilon_{0}^{\prime 2}}{\left[1-\epsilon_{0}^{\prime}\right]^{4}} \frac{\delta_{0}^{\prime 4}}{g^{2}}\left|\frac{\delta_{n_{l_{1}}}}{\gamma_{n_{l_{1}}}}+\frac{\alpha_{n_{l_{2}}}}{\gamma_{n_{l_{2}}}}\right|^{-2}
$$

Let $d_{l}$ be the distance from an isometric circle at level $l$ to the center of the configuration. If the isometric circles at level $l+l_{0}$ are labelled by the index $\mathrm{j}$, a sum of inverse squares of distances from $I_{T_{n_{l_{1}}}}$ to $I_{T_{n_{l+l_{0}}}^{(j)}}$ is required by equations (14) and (A.6). 
When $l+l_{0} \gg 1$, this sum may be approximated by the integral

$$
2 \int \frac{d x}{\left[d_{l+l_{0}}^{2}+d_{l}^{2}-2 d_{l} d_{l+l_{0}} \cos \left(\frac{x \pi}{3\left(l+l_{0}-1\right)}\right)\right]}=\frac{6\left(l+l_{0}-1\right)}{d_{l+l_{0}}^{2}-d_{l}^{2}}
$$

so that the following bound

$$
\begin{aligned}
& 18 \frac{\epsilon_{0}^{\prime 2}}{\left[1-\epsilon_{0}^{\prime}\right]^{4}} \frac{\delta_{0}^{\prime 4}}{\delta_{0}^{4}} \sum_{l=2}^{\left[\frac{1}{2}+\frac{1}{6} \sqrt{9+24 g}\right]}[l-1]\left[1+\frac{1}{2 l}+\sum_{\substack{l_{0}=-l+2 \\
l_{0} \neq 0}}^{\left\{2 \frac{\delta_{0}^{\prime}}{\delta_{0}}\left[1+\frac{2 \epsilon_{0}^{\prime \frac{1}{2}}}{1-\epsilon_{0}^{\prime}}\right]\right\}}\left|\frac{1}{l_{0}}+\frac{1}{2 l+l_{0}}\right|\right] \\
& +18 \frac{\epsilon_{0}^{\prime 2}}{\left[1-\epsilon_{0}^{\prime}\right]^{4}} \frac{\delta_{0}^{\prime 4}}{\delta_{0}^{4}} \sum_{l=2}^{\left[\frac{1}{2}+\frac{1}{6} \sqrt{9+24 g}\right]}[l-1] \\
& \sum^{\left[-\frac{1}{2}+\frac{1}{6} \sqrt{9+24 g}\right]-1}\left[l_{0}-2 \frac{\delta_{0}^{\prime}}{\delta_{0}}\left[1+\frac{2 \epsilon_{0}^{\prime \frac{1}{2}}}{1-\epsilon_{0}^{\prime}}\right]\right]^{-2}\left[\frac{1}{l_{0}}+\frac{1}{2 l+l_{0}}\right] \\
& l_{0}=\left\{2 \frac{\delta_{0}^{\prime}}{\delta_{0}}\left[1+\frac{2 \epsilon_{0}^{\prime \frac{1}{2}}}{1-\epsilon_{0}^{\prime}}\right]\right\}+1
\end{aligned}
$$

is obtained as $d_{l} \geq[l-1] \frac{\delta_{0}}{\sqrt{g}}$. For large $\mathrm{g}$, the sum grows as $\mathrm{O}(\mathrm{g})$, and since the higher-order terms may be bounded similarly, so that the entire sum $\sum_{\alpha}{ }^{\prime}\left|K_{\alpha}\right|$ is bounded above by a function that increases only linearly with the genus as $g \rightarrow \infty$.

For the third category of isometric circles, the estimates can be found for each of the sums in equation (15). The upper bound for $\sum_{\alpha}{ }^{\prime}\left|K_{\alpha}\right|$ would be affected by a change in the range of distances $\left|\xi_{1 n}-\xi_{2 n}\right|, n=1, \ldots, g$. When $V_{\tilde{\alpha}}=T_{n_{l_{1}}}^{ \pm 1} T_{n_{l_{2}}}^{ \pm 1}$, the distances $\left|\xi_{1 \tilde{\alpha}}+\frac{\delta_{\tilde{\alpha}}}{\gamma_{\tilde{\alpha}}}\right|$ are not correlated as closely with the distances $\left|\frac{\delta_{T_{n_{l_{1}}}^{ \pm 1}}}{\gamma_{T_{n_{l_{1}}}^{ \pm 1}}}+\frac{\alpha_{T_{n_{l_{2}}}^{ \pm 1}}}{\gamma_{T_{n_{n_{2}}}^{ \pm 1}}}\right|$. Suppose that $j_{1}$ and $j_{2}$ index the isometric circles $\left\{I_{T_{n_{l_{1}, j_{1}}}}, j_{1}=0,1, \ldots, 6(l-1)-1\right\}$ at level $l$ and the circles $\left\{I_{T_{n_{l_{2}, j_{2}}}^{-1}}, j_{2}=0,1, \ldots, 6\left(l+l_{0}-1\right)-1\right\}$ at level $l+l_{0}$. When $V_{\tilde{\alpha}}=T_{n_{l_{1}}} T_{n_{l_{2}}}$,

$$
\left|\frac{\delta_{T_{n_{l_{1}}}^{\left(j_{1}\right)}}}{\gamma_{T_{n_{l_{1}}}^{\left(j_{1}\right)}}}+\frac{\alpha_{T_{n_{l_{2}}}^{\left(j_{2}\right)}}}{\gamma_{T_{n_{l_{2}}}^{\left(j_{2}\right)}}}\right|^{-2} \leq\left[d_{l+l_{0}}^{2}+d_{l}^{2}-2 d_{l} d_{l+l_{0}} \cos \left(\frac{i\left(j_{1}, j_{2}\right) \pi}{3\left(l+l_{0}-1\right)}\right)\right]^{-1}
$$

where $i\left(j_{1}, j_{2}\right)=0,1, \ldots, 6\left(l+l_{0}-1\right)-1$, but $d\left(I_{T_{n_{l_{1}, j_{1}}}^{-1}}, I_{T_{n_{l_{2}, j_{2}}}}\right)$ can range from $\frac{\delta_{0}}{\sqrt{g}}$ to $2 \frac{\delta_{0}^{\prime}}{g^{q}}+\left|\frac{\delta_{T_{n_{1}}^{\left(j_{1}\right)}}}{\gamma_{T_{n_{l_{1}}}^{\left(j_{1}\right)}}}+\frac{\alpha_{T_{n_{2}}^{\left(j_{2}\right)}}}{\gamma_{T_{n_{l_{2}}}^{\left(j_{2}\right)}}}\right|$. When $\left|l_{0}\right|$ lies in the range $\left[0,2 \frac{\delta_{0}^{\prime}}{\delta_{0}} g^{\frac{1}{2}-q}\right],\left|\xi_{1 \alpha}+\frac{\delta_{\alpha}}{\gamma_{\alpha}}\right|$ can assume its 
minimum value $\frac{\delta_{0}}{\sqrt{g}}$. Beyond this range

$$
\left|\xi_{1 \alpha}+\frac{\delta_{\alpha}}{\gamma_{\alpha}}\right| \geq\left|l_{0}\right| \frac{\delta_{0}}{\sqrt{g}}-2 \frac{\delta_{0}^{\prime}}{g^{q}}\left[1+\frac{2 \epsilon_{0}^{\prime \frac{1}{2}}}{\left(1-\frac{\epsilon_{0}^{\prime}}{g^{1-2 q}}\right)}\right]
$$

Replacing the limit of the first sum over $l_{0}$ in equation (A.8) by $\left\{2 \frac{\delta_{0}^{\prime}}{\delta_{0}} g^{\frac{1}{2}-q}\right\}$ leads to a bound of the form $6 \frac{\epsilon_{0}^{\prime 2}}{\left(1-\epsilon_{0}^{\prime}\right)^{4}} \frac{\delta_{0}^{\prime 4}}{\delta_{0}^{4}}\left(\frac{1}{2}-q\right) g \ln g$ for $\sum_{\alpha}{ }^{\prime}\left|K_{\alpha}\right|$. This modification would be sufficient to obtain an upper bound for the primitive-element products that increases at a factorial rate with respect to the genus, and it is necessary to refine the estimate of the sum. By considering the densest packing of $6\left(l+l_{0}-1\right)$ circles $\left\{I_{T_{n_{l_{2}}}}\right\}$ about $I_{T_{n_{l_{1}, j_{1}}}^{-1}}$, one finds that the distances $\left[d\left(I_{T_{n_{l_{1}, j_{1}}^{-1}}},\left\{I_{T_{n_{l_{2}, j_{2}}}}\right\}\right)\right]^{-2}$ range from $\frac{\delta_{0}}{\sqrt{g}}$ to $\left[-\frac{1}{2}+\frac{1}{2} \sqrt{1+8\left(l+l_{0}-1\right)}\right] \frac{\delta_{0}}{\sqrt{g}}$. Using the average value of $\left[d\left(I_{T_{n_{l_{1}, j_{1}}}^{-1}},\left\{I_{T_{n_{l_{2}, j_{2}}}}\right\}\right)\right]^{-2}$, which is approximately $\frac{g}{2 \delta_{0}^{2}} \frac{\ln \left(l+l_{0}-1\right)}{l+l_{0}-1}$, it follows that the sum over elements $V_{\tilde{\alpha}}=T_{n_{l_{1}}}^{ \pm 1} T_{n_{l_{2}}}^{ \pm 1}$ in the upper bound for $\sum_{\alpha}{ }^{\prime}\left|K_{\alpha}\right|$ will be less than

$$
\begin{gathered}
\epsilon_{0}^{\prime 2}\left[1-\frac{\epsilon_{0}^{\prime}}{g^{1-2 q}}\right]^{-4} \frac{\delta_{0}^{\prime 4}}{\delta_{0}^{4}} \\
{\left[\sum_{l=2}^{\left[\frac{1}{2}+\frac{1}{6} \sqrt{9+24 g}\right]} 18[l-1]\left[1+\frac{1}{2 l}+\frac{1}{2} \sum_{\substack{l_{0}=-l+2 \\
l_{0} \neq 0}}^{\left\{2 \frac{\delta_{0}^{\prime}}{\delta_{0}} g^{\frac{1}{2}-q}\right\}} \frac{\ln \left(l+l_{0}-1\right)}{l+l_{0}-1}\left|\frac{1}{l_{0}}+\frac{1}{2 l+l_{0}}\right|\right]\right.} \\
\left.+\sum_{l=2}^{\left[\frac{1}{2}+\frac{1}{6} \sqrt{9+24 g}\right]} 18[l-1] \sum_{l_{0}=\left\{2 \frac{\delta_{0}^{\prime}}{\delta_{0}} g^{\frac{1}{2}-q}\right\}+1}^{\left[-\frac{1}{6} \sqrt{9+24 g}\right]-1}\left[l_{0}-2 \frac{\delta_{0}^{\prime}}{\delta_{0}} g^{\frac{1}{2}-q}\right]^{-2}\left[\frac{1}{l_{0}}+\frac{1}{2 l+l_{0}}\right]\right]
\end{gathered}
$$

Evaluation of the factor $\left|\xi_{1 \tilde{\alpha}}+\frac{\delta_{\tilde{\alpha}}}{\gamma_{\tilde{\alpha}}}\right|$ implies that the contribution of the generators $\left\{I_{\left.T_{n_{l}}^{ \pm 1}\right\}}\right.$ to $\sum_{\alpha}{ }^{\prime}\left|K_{\alpha}\right|$ will be of order $O\left(g^{2 q}\right)$, indicating, together with equation (A.11) that the sum will be bounded by a linearly increasing function of the genus. The primitive-element product factors should not alter significantly the growth of the bound obtained after integration over the multipliers and fixed-points. 


\section{References}

[1] D. Friedan and S. Shenker, Phys. Lett. $\underline{B 175}$ (1986) 287

D. Friedan and S. Shenker, Nucl. Phys. $\underline{B 281}$ (1987) 509

[2] D. J. Gross 'Non-Perturbative String Theory' in Random Sur faces and Quantum

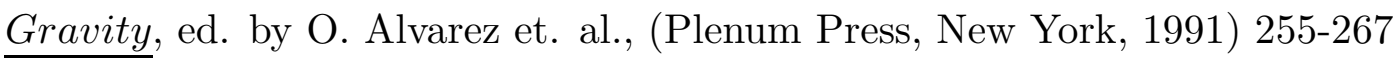

[3] D. J. Gross and V. Periwal, Phys. Rev. Lett. $\underline{60}$ (1988) 2105

[4] S. Mandelstam, 'The Interacting String Picture and Functional Integration' Proceedings of the Workshop on Unified String Theories, ITP-Santa Barbara, ed. by M. Green and D. Gross (Singapore: World Scientific, 1986) 46-102

[5] S. Davis, ICTP preprint (1992) IC/92/431

[6] S. Davis, University of Cambridge preprint (1994) DAMTP-R/94/1

[7] C. L. Siegel, Topics in Complex Function Theory, Vol.3 (New York: Wiley, 1973)

[8] J. L. Petersen, K. O. Roland and J. R. Sidenius, Phys. Lett. B205 (1988) 262-266 J. L. Petersen and J. R. Sidenius, Nucl. Phys. B301 (1988) 247-266 K. O. Roland, Nucl. Phys. B313 (1989) 432-446

[9] S. Davis, Class. Quantum Gravity $\underline{7}$ (1990) 1887-1893

[10] E. D'Hoker and D. H. Phong, Rev. Mod. Phys. Vol. 므, No. 4 (1988) 917-1065

[11] B. Bollobas, J. Lond. Math. $\underline{26}$ (1982) 201-206

[12] A. A. Tseytlin, Int. J. Mod. Phys. A Vol. $\underline{5}$, No. 4 (1990) 589-658

[13] D. Gepner and E. Witten, Nucl. Phys. $\underline{B 278}$ (1986) 493-549 